\title{
DITHIOCARBAMATE SUBSTITUTED PHENOTHIAZINE DERIVATIVES: IN SILICO EXPERIMENTS, SYNTHESIS, AND BIOLOGICAL EVALUATION
}

\author{
ANUSHA KOTHA ${ }^{1,2}$, MUNI SIREESHA S. ${ }^{2}$, ASHMA MD ${ }^{1,2}$, JYOTHI VEMURI ${ }^{3}$, SARITHA JYOSTNA T. ${ }^{2 *}$ \\ 1Department of Pharmaceutical Chemistry, Faculty of Pharmacy, Nalla Narasimha Reddy Educational Society, Hyderabad, Telangana, India, \\ ${ }^{2}$ Department of Pharmaceutical Chemistry, Faculty of Pharmacy, Sarojini Naidu Vanita Pharmacy Mahavidyalaya, Hyderabad, Telangana, India, \\ ${ }^{3}$ Department of Pharmacognosy, Faculty of Pharmacy, Sarojini Naidu Vanita Pharmacy Mahavidyalaya, Hyderabad, Telangana, India \\ *Email: sarithavanita13@gmail.com
}

Received: 17 May 2021, Revised and Accepted: 18 Aug 2021

\section{ABSTRACT}

Objective: The present study was designed to study the anticancer activity of a series of novel analogs of phenothiazine with dithiocarbamate as a side chain.

Methods: A novel series of derivatives containing dithiocarbamate as a side chain at the tenth position of phenothiazine nucleus were synthesized, characterized by spectral analysis, and evaluated for their antimitotic and antioxidant activity using germinated Bengal gram seeds and 2,2diphenyl-1-picrylhydrazyl (DPPH) method, respectively. A quantitative estimate of drug-likeness was also performed, which calculated the molecular properties and screened the molecules based on drug-likeness rules. Further, molecular docking study was performed for finding the binding affinity with tubulin protein to rationalize their anticancer activity.

Results: The results revealed that the antioxidant activity of compounds $3 \mathrm{e}, 3 \mathrm{~g}, 3 \mathrm{i}, 3 \mathrm{j}$ and standard Ascorbic acid were $10 \mathrm{mmol}, 14 \mathrm{mmol}, 16 \mathrm{mmol}$, $16 \mathrm{mmol}$ and $35 \mathrm{mmol}$, respectively. Further compounds $3 \mathrm{e}, 3 \mathrm{~g}$, 3h and 3i have shown promising antimitotic activity. Compound $3 \mathrm{i}(-9 \mathrm{~K}$. Cal/mol) showed the highest binding energies towards tubulin protein when compared to standard drug colchicine (-8.6 K. Cal/mol). Among all, compound $3 \mathrm{i}$ showed promising antimitotic and antioxidant activity, which correlated with insilico docking studies.

Conclusion: Dithiocarbamate substituted phenothiazine derivatives proved to be encouraging leads as tubulin inhibitors.

Keywords: Dithiocarbamate, Molsoft, Molinspiration, Osiris, Pkcsm, Auto dock vina and antimitotic

(c) 2021 The Authors. Published by Innovare Academic Sciences Pvt Ltd. This is an open access article under the CC BY license (https://creativecommons.org/licenses/by/4.0/) DOI: https://dx.doi.org/10.22159/ijpps.2021v13i10.41882. Journal homepage: https://innovareacademics.in/journals/index.php/ijpps.

\section{INTRODUCTION}

In recent years, the design and synthesis of novel bioactive compounds gained significant applications in the pharmaceutical industries. Phenothiazine ring systems are of considerable interest as it is a core structure in various synthetic pharmaceuticals displaying a broad spectrum of biological activities including tranquilizers, antiinflammatory, antimalarial, anti-psychotropic, antimicrobial, antitubercular, antitumor, antihistamine and analgesic properties [13]. Moreover, phenothiazine derivatives having an acyl side chain playing a crucial role in anticancer activity. Other side dithiocarbamates have exhibited colossal pharmacological activities; especially the sulfur atom in dithiocarbamate possesses strong nucleophilic and redox properties. Several literature reports indicate inclusion of dithiocarbamate as a linker or side chain in active pharmacophore improves the overall biological profile [4-7]. Fig. 1 represents the natural products and marketed drugs mostly contain dithiocarbamate moiety [8]. Inspired by these findings, we designed novel dithiocarbamate substituted $\mathrm{N}$-acyl phenothiazine derivatives as anticancer agents.

ComputerAided Drug Design (CADD) is a widely used term that represents computational tools, resources for the storage, management, analysis, and modeling of compounds [9]. An ideal computational method for lead discovery should be able to generate structurally diverse leads and should give an estimate of binding affinities that would correlate with experimental values. The molecular structure is based on physicochemical, drug metabolism, pharmacokinetics (DMPK), and toxicity properties [10]. High oral bioavailability is a vital consideration for the development of bioactive molecules as therapeutic agents. Therefore, the bioavailability-related prediction of properties such as solubility, lipophilicity, good drug absorption, low polar surface area, the sum of hydrogen bond donors and acceptors, molecular weight, partition coefficient $(\log P)$ are vital before actual synthesis to reduce the chemical expenses and precious time. The molecular properties of compounds can be calculated using Molinspiration [11], Molsoft [12], Osiris [13], pKCSM [14], and Swiss Absorption Distribution Metabolism and Excretion (ADME) [15] software which help to reduce cost, late-stage failures and hasten the successful development of new molecular moieties.

Molecular docking may be defined as an optimization problem, which would outline the best-fit orientation of a ligand that binds to a particular protein of interest and is used to expect the structure of the intermolecular complex formed between two or more molecules.

The current study incorporates the use of insilico molecular modeling tool Auto dock Vina [16]. The receptor grid that was generated will helps in locating the protein active site and preparing the grid for the ligands to be docked in the shape and properties of the receptor are represented on a grid by many different sets of fields that provide progressively more precise scoring of the ligand poses. The binding energies of mentioned analogs further clarify the design of potential drug candidates against tubulin protein.

\section{MATERIALS AND METHODS}

All chemicals were purchased from Aldrich and Merck and were used without further purification. The Melting points were obtained on the Lab India Digital Melting Point instrument and are uncorrected. Infrared spectra were recorded on the ALPHA Bruker instrument and values are given in $\mathrm{cm}^{-1} .{ }^{1} \mathrm{H}$ NMR spectra were recorded in $\mathrm{CDCl}_{3}$ on a Bruker Ux-NMR instrument using methyl silane $\left(\mathrm{Me}_{4} \mathrm{Si}\right)$ as the internal standard. High-Resolution Mass Spectroscopy (HRMS) was recorded on maXis 10138. Each reaction was monitored by using an appropriate solvent system, which was selected by trial and error method on Pre-coated Thin Layer Chromatography (TLC) plates $(0.25 \mathrm{~mm}$ silica gel) were obtained from E. Merck and visualized with Ultra Violet (U. V) light. Column chromatography was performed on Silica gel 60-120 mesh (Merck) using commercially available petroleum ether and ethyl acetate. 


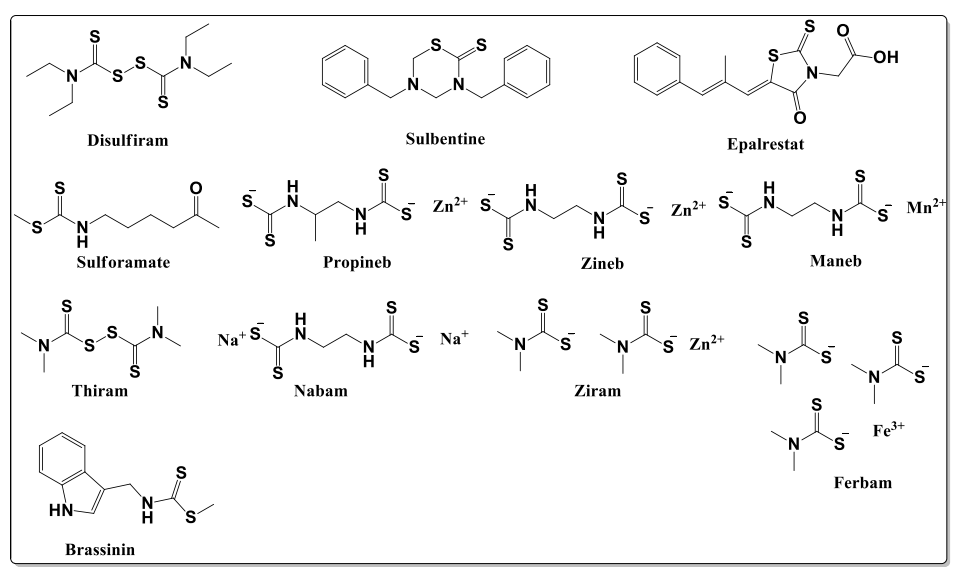

Fig. 1: Natural products and marketed drugs containing dithiocarbamate moiety

Antioxidant activity of the synthesized compounds was performed by the 2,2-diphenyl-1-picrylhydrazyl (DPPH) radical scavenging method [17]. The experiment was carried out at the concentration of $5,10,15$, $20,25, \mu \mathrm{g} / \mathrm{ml}$ in methanol, using ascorbic acid as standard. Results are shown in table 3 and expressed as mmol. The compound 3e (benzyl derivative), showed the best potency, while compound 3g (pyrrolidine derivative) 3i (methyl piperazine derivative), and 3j (ethyl piperazine) showed a slight reduction of antioxidant activity. The compound $3 \mathrm{~h}$ (piperidine derivative) was equipotent with the standard ascorbic acid. The remaining all compounds showed weak activity.

The title compounds 3a-k were screened for their in vitro antimitotic activity [18] using germinated Bengal gram seeds and methanol in a control group

The molecular docking was performed using AUTO DOCK Vina software installed on a single machine running on an Intel Core i53317U CPU @ 1.70 GHz Processor with 6 GB RAM and Windows7 with 64-bit Operating System [19]. With a known biological target named tubulin (PDB ID:-1SA0) for phenothiazine substituted dithiocarbamate compounds. A grid was generated around the co-crystallized ligand. The coordinates $(\mathrm{x}=116.98, \mathrm{y}=90.11, \mathrm{z}=8.392)$ were generated with the help of MGL Tools and Pharmit: interactive exploration of chemical space (http://pharmit. csb. pitt. edu/). Prepared pdbqt files for both target and ligands. The created inhouse batch file of ligands and target and docking was performed in the absence of water molecules for all
12 molecules (11+1 standard drug). The molecules were analyzed after docking and visualized in the discovery studio for the interactions with the active site amino acids [20].

\section{Synthetic procedures}

Dithiocarbamate substituted $\mathrm{N}$-acyl phenothiazine-derivatives were synthesized in two steps as shown in scheme 1. In the first step, Phenothiazine (1 equiv) in the presence of dry benzene $20 \mathrm{ml}$, treated with chloroacetylchloride ( 2 equiv) at $0-5^{\circ}$ and refluxed for 3-4 h at $50-60{ }^{\circ} \mathrm{C}$ temperature.

In the second step, an equimolar mixture of appropriate amine and anhydrous potassium carbonate in dimethylformamide was stirred at room temperature for $5 \mathrm{~min}$, and then carbon disulfide ( 2 equiv) was added. The reaction mixture was stirred for an additional 20 min, and then appropriate 2-chloro-1-(10H-phenothiazin-10yl)ethan-1-one (1 equiv) was added. Stirring was continued at room temperature until the reaction was completed as monitored by Thin Layer Chromatography (TLC). The mixture was poured into cold water, extracted with ethyl acetate $(3 \times 30 \mathrm{ml})$; the organic phase was washed once more with water and dried with sodium sulfate, and filtered. The solvent was evaporated under reduced pressure and the resultant residue was purified by chromatography over silica gel using a mixture of petroleum ether and ethyl acetate as a solvent to give the desired compounds $3 \mathrm{a}-\mathrm{k}$.

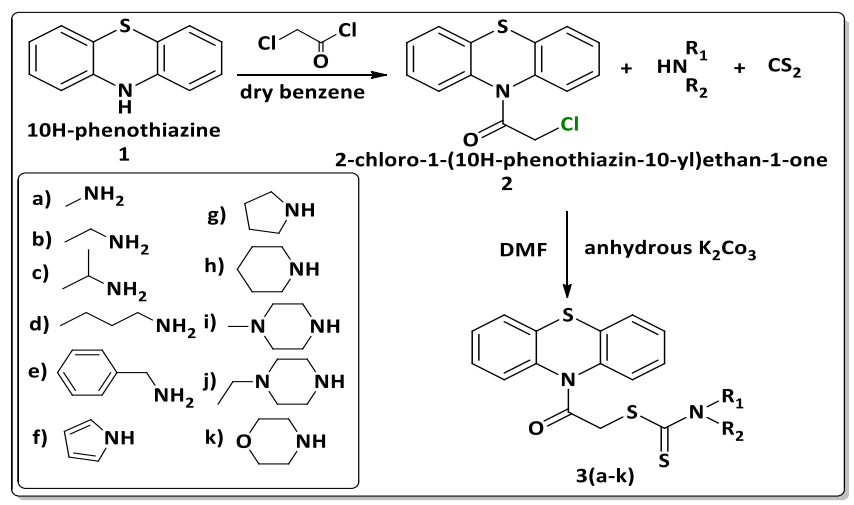

Scheme 1: Synthesis of dithiocarbamate substituted N-acyl phenothiazine derivatives

\section{Synthesis of target derivatives 3a-3k}

Synthesis of 2-oxo-2-(10H-phenothiazin-10-yl) ethylmethylcarbamodithioate: (3a)

Yield (62.5\%); MP 180-182 ${ }^{\circ} \mathrm{C}$; IR(FT-IR-cm ${ }^{-1}$ ) 3334.59(N-H), 3067.71 (aromatic C-H), 2913.27 (aliphatic C-H), $1692.65(\mathrm{C}=0), 1592.99$ (aromatic C=C),1442.20 (C=S), $\left.1302.40(\mathrm{C}-\mathrm{N}) ;{ }^{1} \mathrm{H} \mathrm{NMR}^{\mathrm{C}} \mathrm{CDCl}_{3}-400 \mathrm{MHz}\right]$ $\delta(\mathrm{ppm}) 2.42\left(\mathrm{~s}, 3 \mathrm{H}, \mathrm{CH}_{3}\right), 4.27\left(\mathrm{~s}, 2 \mathrm{H}, \mathrm{CH}_{2}\right), 6.47-7.27$ (m,8H,Ar-H); HRMS calculated for $\mathrm{C}_{16} \mathrm{H}_{14} \mathrm{~N}_{2} \mathrm{~S}_{3} \mathrm{O}$ [M+1]: 346.03, found: 346.01 .

Synthesis of 2-oxo-2-(10H-phenothiazin-10-yl) ethyl ethylcarbamodithioate: (3b)

Yield (70.5\%); MP 182-184 ${ }^{\circ} \mathrm{C}$; IR(FT-IR- $\left.\mathrm{cm}^{-1}\right) \quad 3335.77(\mathrm{~N}-\mathrm{H})$, 3057.05 (aromatic C-H), 2923.06 (aliphatic C-H), 1643.94 (C=O), 
1567.02 (aromatic C=C), $1442.28 \quad(\mathrm{C}=\mathrm{S}), \quad 1303.17 \quad(\mathrm{C}-\mathrm{N}) ;{ }^{1} \mathrm{H}$ NMR[CDCl $3-400 \mathrm{MHz}] \delta(\mathrm{ppm}) 2.25-2.45\left(\mathrm{t}, 3 \mathrm{H}, \mathrm{CH}_{2} \mathrm{CH}_{3}\right), 2.75-2.95(\mathrm{q}$, $\left.2 \mathrm{H}, \mathrm{CH}_{2} \mathrm{CH}_{3}\right), 3.51\left(\mathrm{~s}, 2 \mathrm{H}, \mathrm{CH}_{2}\right), 6.45-7.0(\mathrm{~m}, 8 \mathrm{H}, \mathrm{Ar}-\mathrm{H}) ; \mathrm{HRMS}$ calculated for $\mathrm{C}_{17} \mathrm{H}_{16} \mathrm{~N}_{2} \mathrm{~S}_{3} \mathrm{O}$ [M+1]: 360.04 , found: 360.03 .

Synthesis of 2-oxo-2-(10H-phenothiazin-10-yl) ethyl propan-2ylcarbamodithioate: $(3 c)$

Yield (75\%); MP 180-182 ${ }^{\circ} \mathrm{C}$; IR(FT-IR- $\mathrm{cm}^{-1}$ ) $3337.75(\mathrm{~N}-\mathrm{H})$, 3031.56 (aromatic C-H), 2948.49 (aliphatic C-H), 1644.77(C=0), 1592.55(aromatic C=C), $1442.01 \quad(\mathrm{C}=\mathrm{S}), 1302.35(\mathrm{C}-\mathrm{N}) ;{ }^{1} \mathrm{H}$ $\mathrm{NMR}\left[\mathrm{CDCl}_{3}-400 \mathrm{MHz}\right] \quad \delta(\mathrm{ppm}) 1.53-1.56 \quad\left(\mathrm{~d}, 6 \mathrm{H}, 2 \mathrm{CH}_{3}\right), \quad 3.33-3.39$ $(\mathrm{m}, 1 \mathrm{H}, \mathrm{CH}), 4.27\left(\mathrm{~s}, 2 \mathrm{H}, \mathrm{CH}_{2}\right), 5.82(\mathrm{~s}, 1 \mathrm{H}, \mathrm{NH}), 26.55-7.26(\mathrm{~m}, 8 \mathrm{H}$, Ar-H); HRMS calculated for $\mathrm{C}_{18} \mathrm{H}_{18} \mathrm{~N}_{2} \mathrm{~S}_{3} \mathrm{O}$ [M+1]: 374.06, found: 374.08 .

Synthesis of 2-oxo-2-(10H-phenothiazin-10-yl) ethyl butylcarbamodithioate: (3d)

Yield (68\%); MP 184-186 ${ }^{\circ} \mathrm{C}$; IR(FT-IR-cm $\left.{ }^{-1}\right) 3338.64(\mathrm{~N}-\mathrm{H})$, 3042.65 (aromatic C-H), 2956.49 (aliphatic C-H), $1641.06(\mathrm{C}=\mathrm{O})$, 1593.37 (aromatic C=C),1441.92 (C=S), 1302.45(C-N); ${ }^{1} \mathrm{H}$ NMR[CDCl $3-400 \mathrm{MHz}] \quad \delta(\mathrm{ppm}) 1.72-1.79 \quad(\mathrm{t}, 3 \mathrm{H}, \quad \mathrm{NH}$ $\mathrm{CH}_{2} \mathrm{CH}_{2} \mathrm{CH}_{2} \mathrm{CH}_{3}$ ), 2.52-2.59 (m,4H, NH CH${ }_{2} \mathrm{CH}_{2} \mathrm{CH}_{2} \mathrm{CH}_{3}$ ), 3.15-3.20 $\left(\mathrm{t}, 2 \mathrm{H}, \mathrm{NH} \quad \mathrm{CH}_{2} \mathrm{CH}_{2} \mathrm{CH}_{2} \mathrm{CH}_{3}\right), \quad 4.21 \quad\left(\mathrm{~s}, 2 \mathrm{H}, \mathrm{CH}_{2} \mathrm{~S}\right), \mathrm{S} 7.32-7.82$ $(\mathrm{m}, 8 \mathrm{H}, \mathrm{ArH}), 9.46(\mathrm{~s}, 1 \mathrm{H}, \mathrm{NH})$; HRMS calculated for $\mathrm{C}_{19} \mathrm{H}_{20} \mathrm{~N}_{2} \mathrm{~S}_{3} \mathrm{O}$ $[\mathrm{M}+1]$ : 388.09, found: 388.06 .

Synthesis of 2-oxo-2-(10H-phenothiazin-10-yl) ethyl benzylcarbamodithioate: (3e)

Yield (75\%); MP 178-180 ㄷ; IR(FT-IR-cm ${ }^{-1}$ ) 3336.49 (N-H),3022.04 (aromatic C-H), 2914.81 (aliphatic $\mathrm{C}-\mathrm{H}$ ), 1668.87 (C=0), 1567.46 (aromatic C=C), $1441.60(\mathrm{C}=\mathrm{S}), 1301.82(\mathrm{C}-\mathrm{N})$; ${ }^{1} \mathrm{H} \quad \mathrm{NMR}\left[\mathrm{CDCl}_{3}\right.$ $400 \mathrm{MHz}] \delta(\mathrm{ppm}) 2.24\left(\mathrm{~s}, 2 \mathrm{H}, \mathrm{CH}_{2}\right), 4.21\left(\mathrm{~s}, 2 \mathrm{H}, \mathrm{CH}_{2}\right), 5.81(\mathrm{~s}, 1 \mathrm{H}, \mathrm{NH})$, 6.54-7.26 (m,13H,ArH); HRMS calculated for $\mathrm{C}_{22} \mathrm{H}_{18} \mathrm{~N}_{2} \mathrm{~S}_{3} \mathrm{O}$ [M+1]: 422.06, found: 422.04 .

Synthesis of 2-oxo-2-(10H-phenothiazin-10-yl) ethyl $1 \mathrm{H}$ pyrrole-1-carbodithioate: (3f)

Yield (65.5\%); MP $\quad 176-178 \quad{ }^{\circ} \mathrm{C} ; \quad$ IR(FT-IR-cm $\left.{ }^{-1}\right) \quad 3053.50$ (Aromatic C-H), 2915.05 (Aliphatic C-H), $1691.43(\mathrm{C}=\mathrm{O}), 1421.31$ $(\mathrm{C}=\mathrm{S}), 1236.34(\mathrm{C}-\mathrm{N}) ;{ }^{1} \mathrm{H}$ NMR $\left[\mathrm{CDCl}_{3}-400 \mathrm{MHz}\right] \delta(\mathrm{ppm}) 2.09-2.12$ (t, 2H, Pyrrole) 3.15-3.24 (d, 2H, pyrrole), $4.14\left(\mathrm{~s}, 2 \mathrm{H}, \mathrm{CH}_{2} \mathrm{~S}\right)$, ,7.27$7.82(\mathrm{~m}, 8 \mathrm{H}, \mathrm{ArH})$; HRMS calculated for $\mathrm{C}_{19} \mathrm{H}_{14} \mathrm{~N}_{2} \mathrm{~S}_{3} \mathrm{O}$ [M+1]: 382.05 , found: 382.02 .

Synthesis of 2-oxo-2-(10H-phenothiazin-10-yl) ethyl pyrrolidine-1-carbodithioate: $(3 \mathrm{~g})$

Yield (76.8\%); MP $180-182{ }^{\circ} \mathrm{C}$; IR(FT-IR-cm ${ }^{-1}$ ) 3070.41 (aromatic CH), 2910.76 (aliphatic C-H), 1682.13 (C=0), 1436.73 (C=S), 1156.87 (C-N); ${ }^{1} \mathrm{H}$ NMR[CDCl $\left.3-400 \mathrm{MHz}\right] \delta(\mathrm{ppm}) 1.25-1.32\left(\mathrm{~m}, 4 \mathrm{H}, \mathrm{N}\left(\mathrm{CH}_{2}\right)_{2}\right.$ $\left(\mathrm{CH}_{2}\right)_{2}$ of pyrolidine), 3.25-3.97 (t, $4 \mathrm{H}, \mathrm{N}\left(\mathrm{CH}_{2}\right)_{2}\left(\mathrm{CH}_{2}\right)_{2}$ of pyrolidine), $4.40\left(\mathrm{~s}, 2 \mathrm{H}, \mathrm{CH}_{2} \mathrm{~S}\right), 7.28-7.82(\mathrm{~m}, 8 \mathrm{H}, \mathrm{Ar} \mathrm{H})$; HRMS calculated for $\mathrm{C}_{19} \mathrm{H}_{18} \mathrm{~N}_{2} \mathrm{~S}_{3} \mathrm{O}$ [M+1]: 386.06 , found: 386.07 .

Synthesis of 2-oxo-2-(10H-phenothiazin-10-yl) ethyl piperidine-1-carbodithioate: $(3 \mathrm{~h})$

Yield (68\%); MP $174-176{ }^{\circ} \mathrm{C}$; IR(FT-IR-cm ${ }^{-1}$ ) 3052.79 (aromatic C$\mathrm{H}), \quad 2945.61$ (aliphatic C-H), 1676.84(C=0), 1426.32(C=S), $1228.72(\mathrm{C}-\mathrm{N}) ;{ }^{1} \mathrm{H}$ NMR[CDCl $\left.3-400 \mathrm{MHz}\right] \delta(\mathrm{ppm}) 4.16\left(\mathrm{~s}, 2 \mathrm{H}, \mathrm{CH}_{2} \mathrm{~S}\right)$, 4.10-4.39 (m,10H,N $\left.\left(\mathrm{CH}_{2}\right)_{5}\right), 7.22-7.67(\mathrm{~m}, 8 \mathrm{H}, \mathrm{ArH})$; HRMS calculated for $\mathrm{C}_{20} \mathrm{H}_{20} \mathrm{~N}_{2} \mathrm{~S}_{3} \mathrm{O}$ [M+1]: 400.07 , found: 400.08 .

Synthesis of 2-oxo-2-(10H-phenothiazin-10-yl) ethyl 4methylpiperazine-1-carbodithioate: (3i)

Yield (72\%); MP 176-178 ${ }^{\circ} \mathrm{C}$; IR(FT-IR-cm $\left.{ }^{-1}\right) 3063.92$ (aromatic CH), 2920.94 (aliphatic C-H), 1685.32 (C=0), 1427.99 (C=S), 1227.43 $(\mathrm{C}-\mathrm{N}) ;{ }^{1} \mathrm{H}$ NMR[CDCl $\left.3-400 \mathrm{MHz}\right] \delta(\mathrm{ppm}) 2.15\left(\mathrm{~s}, 3 \mathrm{H}_{1}, \mathrm{NCH}_{3}\right), 2.25-2.96$ $\left(\mathrm{t}, 4 \mathrm{H}\right.$, piperazine), 3.22-3.83 (t,4H,piperazine), $4.36\left(\mathrm{~s}, 2 \mathrm{H}, \mathrm{CH}_{2} \mathrm{~S}\right)$, 7.11-7.60 (m, 8H,ArH); HRMS calculated for $\mathrm{C}_{20} \mathrm{H}_{21} \mathrm{~N}_{3} \mathrm{~S}_{3} \mathrm{O}$ [M+1]: 415.08, found: 415.07 .
Synthesis of 2-oxo-2-(10H-phenothiazin-10-yl) ethyl 4ethylpiperazine-1-Carbodithioate: (3j)

Yield (79\%); MP 178-180 ${ }^{\circ} \mathrm{C}$; IR(FT-IR-cm ${ }^{-1}$ ) 3079.15 (aromatic CH), 2964.55 (aliphatic C-H), 1676.07 (C=0), 1460.70 (C=S), 1232.03 $(\mathrm{C}-\mathrm{N}) ; \quad{ }^{1} \mathrm{H} \quad \mathrm{NMR}\left[\mathrm{CDCl}_{3}-400 \mathrm{MHz}\right] \quad \delta(\mathrm{ppm}) 1.82-1.89 \quad(\mathrm{t}, 3 \mathrm{H}$, $\left.\mathrm{NCH}_{2} \mathrm{CH}_{3}\right), 2.12-2.18\left(\mathrm{q}, 2 \mathrm{H}, \mathrm{NCH}_{2} \mathrm{CH}_{3}\right), 2.15-2.86$ (t,4H,piperazine), 3.12-3.73(t,4H,piperazine), $3.83\left(\mathrm{~s}, 2 \mathrm{H}, \mathrm{CH}_{2} \mathrm{ArH}\right), 4.46\left(\mathrm{~s}, 2 \mathrm{H}, \mathrm{CH}_{2} \mathrm{~S}\right)$, 7.15-7.62 (m,8H,ArH); HRMS calculated for $\mathrm{C}_{21} \mathrm{H}_{23} \mathrm{~N}_{3} \mathrm{~S}_{3} \mathrm{O}[\mathrm{M}+1]$ : 429.10, found: 429.13 .

Synthesis of 2-oxo-2-(10H-phenothiazin-10-yl) ethyl morpholine-4-carbodithioate: (3k)

Yield (68\%); MP $180-182{ }^{\circ} \mathrm{C}$; IR(FT-IR-cm ${ }^{-1}$ ) 3010.49 (aromatic CH), 2949.70 (aliphatic C-H), 1673.08 (C=0), 1460.37 (C=S), 1228.18 (C-N); ${ }^{1} \mathrm{H}$ NMR $\left[\mathrm{CDCl}_{3}-400 \mathrm{MHz}\right] \delta(\mathrm{ppm}) 3.48-4.42\left(\mathrm{t}, 8 \mathrm{H},\left(\mathrm{CH}_{2}\right)_{4}\right), 3.73$ $\left(\mathrm{s}, 2 \mathrm{H}, \mathrm{CH}_{2} \mathrm{~S}\right), \quad$ 7.23-7.67 (m,8H,ArH); HRMS calculated for $\mathrm{C}_{19} \mathrm{H}_{18} \mathrm{~N}_{2} \mathrm{~S}_{3} \mathrm{O}_{2}[\mathrm{M}+1]$ : 402.07 , found: 402.05 .

\section{RESULTS AND DISCUSSION}

The synthesis of title compounds obtained by reaction of dithiocarbamate with $\mathrm{N}$-(2-chloro-acetyl) phenothiazine in the presence of anhydrous Potassium Carbonate $\left(\mathrm{K}_{2} \mathrm{CO}_{3}\right)$ using various amines as shown in (scheme). The chemical structures of the newly synthesized compounds were characterized by infrared (IR), proton nuclear magnetic resonance ( $\left.{ }^{1} \mathrm{H} \mathrm{NMR}\right)$, and mass analysis.

\section{In silico experiments}

The knowledge of absorption, distribution, metabolism, excretion and toxicity (ADMET) are primary requests for the development of new drugs. Lipinski et al. proposed the rule of five, indicating that orally active drugs should have a molecular weight is $\leq 500, \log p \leq 5$, the number of Hydrogen bond donors is $\leq 5$ and the number of Hydrogen bond acceptors is $\leq 10$ [21]. Additionally, Veber et al. proposed that molecular flexibility indicated by rotatable bond count $\leq 10$ and polar surface area is $\leq 140^{\circ} \mathrm{A}$ are important for oral bioavailability in the rat [22].

In this study Molsoft, Molinspiration, Swiss ADME and Pkcsm were used to evaluate pharmacokinetic parameters of all the compounds shown in table 1. It was found that there is a zero violation of the Lipinski rule, all showed NRTOB between 4 to 7 and TPSA values ranged between 20 to 24 , an indication for oral absorption. The molsoft program was used to evaluate the drug likeliness model score. Compounds showing positive drug likeliness model scores are recognized as drug-like and can behave as drug molecules. All predicted compounds showed drug-like properties.

The pharmacokinetic parameters were performed using swiss ADMET software, Human Intestinal Absorption (HIA), human colon adenocarcinoma $\left(\mathrm{CaCO}_{2}\right)$ permeability coefficient, Blood-brain barrier (BBB) shown in table 2. All compounds showed high HIA values (92.01-96.70\%) indicating very well absorbed and they showed low BBB penetration capacity $(0.153-0.288)$. P-glycoprotein (P-gp) acts as a physiological barrier by ejecting drugs and other compounds out of cells, which was found to be one of the reasons for its resistance to various chemotherapeutics for cancer. Inhibition of p-gp results in better bioavailability of the liable drug. Inhibition of Cyt.450 iso-forms results in drug-drug interactions.

\section{Anti-oxidant activity}

According to the results obtained with the DPPH method, the antioxidant activity was increased when introducing aromatic ring or alicyclic moiety on dithiocarbamate and reduced with aliphatic hydrocarbon.

\section{Anti-mitotic activity}

Results are illustrated in table 3 and expressed as mmol. Among the series compounds, $3 \mathrm{e}$ and $3 \mathrm{~g}$ showed maximum inhibition. The activity was slightly reduced for compounds $3 \mathrm{~h}, 3 \mathrm{i}$, and $3 \mathrm{j}$ the activity was reduced slightly. The remaining all compounds showed moderate to weak antimitotic activity. 
Table 1: Molecular properties of 3 (a-k)

\begin{tabular}{|c|c|c|c|c|c|c|c|c|c|}
\hline Comp & MW & $\mathrm{MP}\left({ }^{\circ} \mathrm{C}\right)$ & $\log P$ & HBD & HBA & Rot B & PSA & DLMs & BBB \\
\hline $3 a$ & 346.03 & $180-182$ & 3.71 & 4 & 1 & 4 & 24.39 & 0.14 & 0.187 \\
\hline $3 b$ & 360.04 & $182-184$ & 4.26 & 4 & 1 & 5 & 23.87 & 0.20 & 0.172 \\
\hline $3 c$ & 374.06 & $180-182$ & 4.60 & 4 & 1 & 5 & 23.22 & 0.34 & 0.197 \\
\hline $3 d$ & 388.07 & $184-186$ & 5.22 & 4 & 1 & 7 & 24.08 & 0.20 & 0.127 \\
\hline $3 e$ & 422.06 & $178-180$ & 5.43 & 4 & 1 & 6 & 23.97 & 0.09 & 0.216 \\
\hline $3 f$ & 382.03 & $176-178$ & 4.59 & 4 & 0 & 4 & 16.90 & 0.02 & 0.211 \\
\hline $3 g$ & 386.06 & $180-182$ & 4.68 & 4 & 0 & 4 & 16.80 & 0.32 & 0.268 \\
\hline $3 \mathrm{~h}$ & 400.07 & $174-176$ & 5.04 & 4 & 0 & 4 & 16.43 & 0.42 & 0.251 \\
\hline $3 \mathrm{i}$ & 415.08 & $176-178$ & 3.78 & 5 & 0 & 4 & 19.91 & 1.19 & 0.288 \\
\hline $3 j$ & 429.10 & $178-180$ & 4.27 & 5 & 0 & 5 & 19.97 & 1.18 & 0.319 \\
\hline $3 \mathrm{k}$ & 402.05 & $180-182$ & 3.73 & 5 & 0 & 4 & 24.34 & 0.22 & 0.153 \\
\hline
\end{tabular}

MW-Molecular weight, HBD-No. of hydrogen bond donors, HBA-No. of hydrogen bond acceptors, Rot B-No. of rotatable bonds, PSA-Polar surface area, DLMS-Drug likeliness model score, BBB-blood-brain barrier.

Table 2: ADME properties of compounds of 3 (a-k)

\begin{tabular}{|c|c|c|c|c|c|c|c|c|c|c|c|c|c|}
\hline \multirow{6}{*}{ Absorption } & & & $3 \mathbf{a}$ & 3b & $3 c$ & 3d & $3 \mathbf{e}$ & 3f & $3 g$ & $3 h$ & $3 \mathbf{i}$ & $3 \mathbf{j}$ & $3 \mathbf{k}$ \\
\hline & \multicolumn{2}{|c|}{$\begin{array}{l}\text { Intestinal } \\
\text { (\% absorbed) }\end{array}$} & 93.475 & 93.056 & 92.954 & 92.017 & 92.223 & $\begin{array}{l}96.76 \\
2\end{array}$ & $\begin{array}{l}92.67 \\
4\end{array}$ & 93.285 & 93.57 & 93.151 & 94.961 \\
\hline & \multicolumn{2}{|c|}{ Substrate } & Yes & Yes & Yes & Yes & Yes & Yes & Yes & Yes & Yes & Yes & Yes \\
\hline & \multicolumn{13}{|c|}{ P-glycoprotein (P-gp) inhibitor } \\
\hline & \multicolumn{2}{|l|}{ P-gp I } & Yes & Yes & Yes & Yes & Yes & Yes & Yes & Yes & Yes & Yes & Yes \\
\hline & \multicolumn{2}{|l|}{ P-gp II } & Yes & Yes & Yes & Yes & Yes & Yes & Yes & Yes & Yes & Yes & Yes \\
\hline \multirow[t]{3}{*}{ Distribution } & \multicolumn{2}{|c|}{$\begin{array}{l}\text { VDss (Human) } \\
\text { (log L/kg) }\end{array}$} & 0.693 & 0.775 & 0.82 & 0.97 & 0.625 & 0.635 & 1.047 & 1.094 & 1.499 & 1.593 & 0.806 \\
\hline & \multicolumn{2}{|c|}{$\begin{array}{l}\text { BBB permeability } \\
(\log B B)\end{array}$} & 0.187 & 0.172 & 0.197 & 0.127 & 0.261 & 0.211 & 0.268 & 0.251 & 0.288 & 0.319 & 0.153 \\
\hline & \multicolumn{2}{|c|}{ CNS permeability } & -1.533 & -1.571 & -1.44 & -1.522 & -0.852 & - & - & -1.494 & -1.812 & -1.85 & -1.539 \\
\hline \multirow[t]{7}{*}{ Metabolism } & \multirow[t]{2}{*}{ Substrate } & CYP2D6 & No & No & No & No & Yes & No & No & No & No & No & No \\
\hline & & CYP3A4 & Yes & Yes & Yes & Yes & Yes & Yes & Yes & Yes & Yes & Yes & Yes \\
\hline & \multirow[t]{5}{*}{ Inhibitor } & CYP1A2 & Yes & Yes & Yes & Yes & Yes & Yes & Yes & Yes & Yes & Yes & Yes \\
\hline & & CYP2C19 & Yes & Yes & Yes & Yes & Yes & Yes & Yes & Yes & No & No & Yes \\
\hline & & CYP2C9 & Yes & Yes & Yes & Yes & Yes & Yes & No & No & No & No & No \\
\hline & & CYP2D6 & No & No & No & No & No & No & No & No & No & No & No \\
\hline & & CYP3A4 & No & No & No & Yes & Yes & Yes & No & No & No & Yes & No \\
\hline \multirow{3}{*}{$\begin{array}{l}\text { Excretion (TC) } \\
\text { Toxicity }\end{array}$} & \multicolumn{2}{|c|}{ Log $\mathrm{ml} / \mathrm{min} / \mathrm{kg}$} & 0.186 & 0.12 & 0.045 & 0.179 & 0.037 & 0.083 & 0.213 & 0.164 & 0.1 & 0.142 & 0.56 \\
\hline & \multirow{2}{*}{\multicolumn{2}{|c|}{ MTD (log mg/kg/day) }} & 0.027 & 0.102 & 0.216 & 0.297 & 0.323 & -0.183 & -0.229 & -0.223 & -0.174 & -0.098 & -0.407 \\
\hline & & & 2.871 & 2.93 & 2.843 & 2.947 & 2.535 & 2.386 & 2.892 & 2.914 & 2.891 & 2.945 & 2.789 \\
\hline
\end{tabular}

$\mathrm{VD}=$ volume of distribution; $\mathrm{MTD}=$ Maximum Tolerated Dose; LD = Lethal Dose; $\mathrm{TC}=$ Total Clearence.

Table 3: Antioxidant and antimitotic activities of 3 (a-k)

\begin{tabular}{|c|c|c|}
\hline Compound & Antioxidant activity $\mathrm{IC}_{50}(\mathrm{mM})$ & Antimitotic activity $\mathrm{IC}_{50}(\mathrm{mM})$ \\
\hline $3 a$ & 64.3 & 19 \\
\hline $3 b$ & 43.1 & 11 \\
\hline $3 c$ & 46 & 6 \\
\hline $3 d$ & 28 & 12 \\
\hline $3 e$ & 10 & 4 \\
\hline $3 f$ & 40 & 14 \\
\hline $3 g$ & 14 & 4 \\
\hline $3 \mathrm{~h}$ & 21 & 5 \\
\hline $3 i$ & 16 & 5 \\
\hline $3 j$ & 16 & 9 \\
\hline $3 \mathrm{k}$ & 35 & 10 \\
\hline Standard & 21 (Ascorbic acid) & \\
\hline
\end{tabular}

\section{Docking}

The synthesized molecules were docked into the same grid and checked for interactions with the receptor active sites and compared with the standard drug colchicine. The docking results are shown in table 4. The compounds were found to have binding energies in the range of-7.50 to $-9.00 \mathrm{Kcal} / \mathrm{mol}$ for tubulin protein. All the compounds docked into the structure were deeply embedded into the hydrophobic pocket. The docking result indicating that $\mathrm{H}$-bond interactions and hydrophobic interactions were important for the inhibition of tubulin protein, as shown in fig. 2. Among 11 compounds, structures $3 \mathrm{f}, 3 \mathrm{i}, 3 \mathrm{j}$ and $3 \mathrm{k}$ were shown equivalent binding energies when compared with colchicine drug-8.7,-9,-8.7,8.6 and-8.6, respectively. Overall results demonstrating, cyclic compounds with two heteroatoms on the dithiocarbamate side chain were important for tubulin inhibition. 
Table 4: Docking interactions of tubulin protein (1SA0) and ligands 3 (a-k)

\begin{tabular}{|c|c|c|c|}
\hline $\begin{array}{l}\text { Compound } \\
\text { Id }\end{array}$ & $\begin{array}{l}\text { Binding energy } \\
\text { (K Cal/mol) }\end{array}$ & Hydrogen bond interactions & Hydrophobic interactions \\
\hline $3 \mathrm{a}$ & -7.5 & LEU B: 248 & ALA A: 180, LYS B: 352, LEU B: 255, ALA B: 250, CYS B: 241 \\
\hline $3 b$ & -7.7 & ASN A: 101 & ALA A: 180, LYS B: 352, LEU B: 248, ALA B: 250, LYS B: 254 \\
\hline $3 c$ & -7.9 & THR A: 179 & $\begin{array}{l}\text { ALA A: } 180, \text { LYS B: 352, LEU B: 255, ALA B: 250, CYS B: 241, ALA B: } 316 \text {, } \\
\text { LEU B: } 248\end{array}$ \\
\hline $3 d$ & -7.5 & THR A: 179, ASN A: 101 & $\begin{array}{l}\text { LEU B: 255,LYS B: } 254, A L A \text { A: } 180, \text { ALA B: } 250, \text { ALA B: 354, LUE B: } 248 \text {, } \\
\text { VAL A: 181, LYS B: } 352\end{array}$ \\
\hline $3 e$ & -8.3 & THR A: 179 & LYS B: 352, CYS B: 241, LEU B: 248, LUE B: 255, ALA B: 250 \\
\hline $3 f$ & -8.7 & & ALA B: 316, ALA B: 354, VAL B: 318, LYS B: 254 \\
\hline $3 g$ & -7.9 & SER A: 178 & $\begin{array}{l}\text { LEU B: 255,LYS B: 254,ALA B: 250, LEU B: 248, CYS B: 241, VAL B: } 318 \text {, } \\
\text { ALA B: 316, ALA B: } 354\end{array}$ \\
\hline $3 \mathrm{~h}$ & -7.7 & ASN B: 350, PRO B: 348. ASN B: 349 & LEU B: 333 \\
\hline $3 \mathrm{i}$ & -9 & GTP A: 600, THR A: 179 & ALA A: 180, ALA B: 250, LEU B: 255, LEU B: 248, LYS B: 352, CYS B: 241 \\
\hline $3 \mathrm{j}$ & -8.7 & ASN A: 101, ALA B: 250 & ALA A: 180, LYS B: 352, LEU B: 255, LEU B: 248, CYS B: 241 \\
\hline $3 \mathrm{k}$ & -8.6 & TYR A: 224 & ALA A: 180, LYS B: 352, LEU B: 248, ALA B: 250, CYS B: 241, LEU B: 255 \\
\hline Colchicine & -8.6 & $\begin{array}{l}\text { LYS B: } 254, \text { ASN A: } 101, \text { THR B: } 353 \\
\text { LEU B: } 248, \text { LYS B: } 352\end{array}$ & ALA B: 354 \\
\hline
\end{tabular}

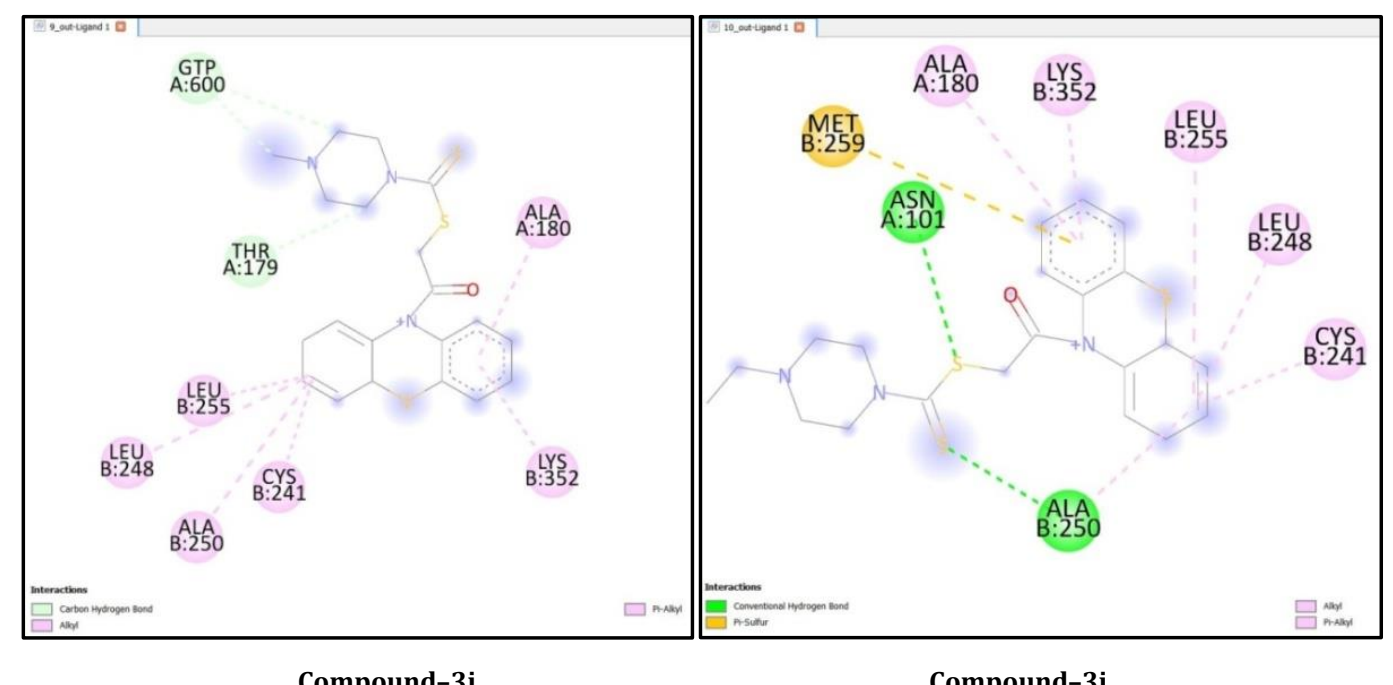

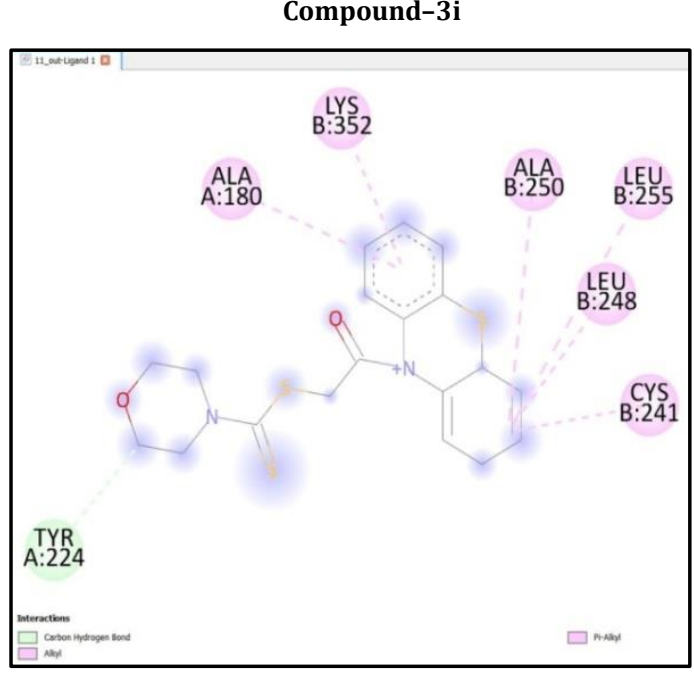

Compound-3k

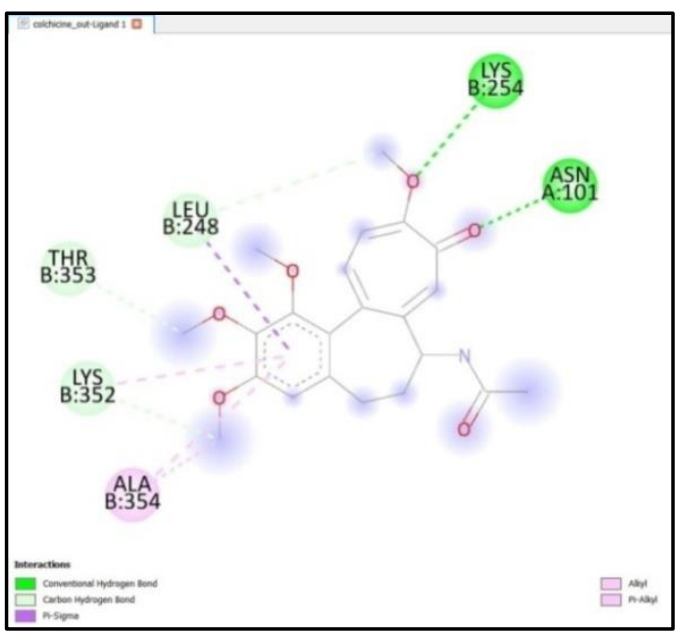

colchicine

Fig. 2: Dock poses of compound 3i, 3j, 3k and colchicine into Tubulin protein (1SA0) showing hydrogen bond interactions (口? Hydrophobic interactions (

\section{CONCLUSION}

In the present study, we designed and synthesized a series of dithiocarbamate substituted phenothiazine derivatives. The derivatives were characterized by IR, ${ }^{1} \mathrm{H}$ NMR, and HRMS. All the derivatives were screened for their antioxidant activity by the DPPH method, antimitotic activity using germinated Bengal gram seeds, and in silico experiments by various software. According to the results compounds $3 \mathrm{e}, 3 \mathrm{~g}, 3 \mathrm{i}$ and $3 \mathrm{j}$ emerged as promising antioxidant agents. Further, compounds $3 \mathrm{e}, 3 \mathrm{~g}, 3 \mathrm{~h}, 3 \mathrm{i}$, and $3 \mathrm{j}$ 
emerged as prominent antimitotic agents. From the in silico studies, all the compounds had drug-like properties and the best hit from auto dock vina are compounds $3 \mathrm{f}, 3 \mathrm{i}, 3 \mathrm{j}$, and $3 \mathrm{k}$ when compared to colchicine, interacted prominently with the binding pocket of protein of tubulin receptor with remarkable hydrogen bonding and hydrophobic interactions. According to the docking scores, we suggest that compounds $3 \mathrm{e}$ are significantly active and this correlates with antioxidant and antimitotic activities. These interesting findings suggested that phenothiazine with dithiocarbamate as a side chain analog can serve as promising leads for inhibition of tubulin protein.

\section{ACKNOWLEDGMENT}

The authors are thankful to the Principal and Management of Sarojini Naidu Vanitha Pharmacy MahaVidhyalaya, Osmania University, Hyderabad, India, for providing research facilities.

\section{FUNDING}

Nil

\section{AUTHORS CONTRIBUTIONS}

The experiment was conducted under the guidance of $T$. SarithaJyostna, KothaAnusha: conduct of experiments, literature collection, and analysis, S. MuniSireesha: Docking studies, Mohammed Ashma: Contribution in biological activity, V. Jyothi: Provided facilities for the experiments

\section{CONFLICT OF INTERESTS}

The authors declare no conflict of interest.

\section{REFERENCES}

1. Ghinet A, Moise IM, Rigo B, Homerin G, Farce A, Dubois J, Bîcu E. Studies on phenothiazines: new microtubule-interacting compounds with phenothiazine A-ring as potent antineoplastic agents. Bioorg Med Chem. 2016;24(10):2307-17. doi: 10.1016/j.bmc.2016.04.001, PMID 27073050.

2. Addla D, Jallapally A, Gurram D, Yogeeswari P, Sriram D, Kantevari S. Rational design, synthesis and antitubercular evaluation of novel 2-(trifluoromethyl)phenothiazine[1,2,3]triazole hybrids. Bioorg Med Chem Lett. 2014;24(1):2336. doi: 10.1016/j.bmcl.2013.11.031.

3. Bansode TN, Shelke JV, Dongre VG. Synthesis and antimicrobial activity of some new $\mathrm{N}$-acyl substituted phenothiazines. Eur J Med Chem. 2009;44(12):5094-8. doi: 10.1016/j.ejmech.2009.07.006, PMID 19651462.

4. Altıntop MD, Sever B, Akalın Çiftçi G, Kucukoglu K, Özdemir A, Soleimani SS, Nadaroglu H, Kaplancıklı ZA. Synthesis and evaluation of new benzodioxole-based dithiocarbamate derivatives as potential anticancer agents and hCA-I and hCA-II inhibitors. Eur J Med Chem. 2017;125:190-6. doi: 10.1016/j.ejmech.2016.09.035.

5. Duan YC, Ma YC, Zhang E, Shi XJ, Wang MM, Ye XW, Liu HM. Design and synthesis of novel 1,2,3-triazole-dithiocarbamate hybrids as potential anticancer agents. Eur J Med Chem. 2013;62:11-9. doi: 10.1016/j.ejmech.2012.12.046, PMID 23353743.
6. Zou Y, Yu S, Li R, Zhao Q, Li X, Wu M, Huang T, Chai X, Hu H, Wu Q. Synthesis, antifungal activities and molecular docking studies of novel2-(2,4-difluorophenyl)-2-hydroxy-3-(1H-1,2,4triazol-1-yl)propyl Dithiocarbamates. Eur J Med Chem. 2014;74:366-74. doi: 10.1016/j.ejmech.2014.01.009.

7. Csomós P, Zupkó I, Réthy B, Fodor L, Falkay G, Bernáth G. Isobrassinin and its analogues: novel types of antiproliferative agents. Bioorg Med Chem Lett. 2006;16(24):6273-6. doi: 10.1016/j.bmcl.2006.09.016.

8. Sangita DS, Akash PS, Shankaraiah N. An insight into medicinal attributes of dithiocarbamates: bird's eye view. Bioorg Chem. 2020;105. PMID 104346.

9. Ooms F. Molecular modeling and computer-aided drug design. Examples of their applications in medicinal chemistry. Curr Med Chem. 2000;7(2):141-58. doi: 10.2174/0929867003375317, PMID 10637360.

10. Raimund M, Han WBM, Bernard T, Hugo K. Drug bioavailability: estimation of solubility, permeability, absorption and bioavailability. Burg Chem. 2008;43:146-7.

11. www.molinspiration.com/cgi-bin/properties. [Last accessed on 10 Apr 2021]

12. www.molsoft.com/mprop/. [Last accessed on 10 Apr 2021]

13. www.organicchemistry.org/prog/peo/OSIRISPropertyExplorer. accessed on 10 Apr 2021]

14. Pires DE, Blundell TL, Ascher DB. pkCSM: Predicting SmallMolecule Pharmacokinetic and Toxicity Properties Using Graph-Based Signatures. J Med Chem. 2015;58(9):4066-72. doi: 10.1021/acs.jmedchem.5b00104, PMID 25860834.

15. swissadme.ch/index.php. [Last accessed on 10 Apr 2021]

16. Trott 0 , Olson AJ. AutoDock Vina: improving the speed and accuracy of docking with a new scoring function, efficient optimization, and multithreading. J Comput Chem. 2010;31(2):455-61. doi: 10.1002/jcc.21334, PMID 19499576.

17. Brand Williams W, Cuvelier ME, Berset C. Use of a free radical method to evaluate antioxidant activity. LWT Food Sci Technol. 1995;28(1):25-30. doi: 10.1016/S0023-6438(95)80008-5.

18. Narsimha Reddy RA. Evaluation of antimitotic activity of indole derivatives on germinating Bengal gram seeds. World J Pharm Life Sci. 2017;3:278-81.

19. Ewing TJA, Kuntz ID. Critical evaluation of search algorithms for automated molecular docking and database screening. J Comput Chem. 1997;18(9):1175-89. doi: 10.1002/(SICI)1096987X(19970715)18:9<1175::AID-JCC6>3.0.CO;2-0.

20. Morris GM, Goodsell DS, Halliday RS, Huey R, Hart WE, Belew RK, Olson AJ. Automated docking using a Lamarckian genetic algorithm and an empirical binding free energy function. J Comput Chem. 1998;19(14):1639-62. doi: 10.1002/(SICI)1096987X(19981115)19:14<1639::AID-JCC10>3.0.CO;2-B.

21. Siwek A, Plech T, Stefańska J, Stączek P, Strzelczyk A. Molecular properties prediction, docking studies, and antimicrobial screening of 1,3,4-thiadiazole and s-triazole derivatives. Curr Comput Aided Drug Des. 2014;10(1):3-14. doi: 10.2174/15734099113096660033, PMID 24138398.

22. Veber DF, Johnson SR, Cheng HY, Smith BR, Ward KW, Kopple KD. Molecular properties that influence the oral bioavailability of drug candidates. J Med Chem. 2002;45(12):2615-23. doi: 10.1021/jm020017n, PMID 12036371. 